Scott, for accomplishing their important task in such a painstaking and skilful fashion.

If we now turn to the contents of this first volume, which covers Newton's youth and his first creative period, during which most of the optical work was done and the new ideas about mathematical analysis and the theory of gravitation took shape, I cannot say that it discloses any striking novelty. Most of the letters and documents had already been published, and the fuller view we now gain by their more complete presentation can only increase our respect for the judgment of the Victorian scholars, Edleston, Rigaud, Brewster, who made the previous selections; it is comforting to realize, in particular, what careful and sagacious use good old Brewster, with all his oddities, could make of his sources. Nevertheless, renewed studies of these sources in their authentic and complete form cannot fail to reveal interesting new details, enlivening the picture of the times and deepening our understanding of the processes of discovery. Especially valuable in the latter respect is the publication of numerous documents illustrating the little-known relations between Newton and Gregory ; it is the editor's great merit to have directed attention to them.

Reading such correspondence, in the chronological order in which it is presented, is like listening to a symphony the various themes of which successively appear and interlace, evoking the hero's growth and deeds against the background of his time and environment. In this respect, the comparison of the Huygens and Newton papers during the same span of their lives (three volumes to one!) illustrates in a most telling fashion the difference between the social classes to which they belonged and which supported and influenced their work. Of Newton's rustic youth scarcely any rocord remains, whereas there is a wealth of documents picturing all the stages of the opulent Huygens children's refined education and their development into sophisticated and slightly snobbish gentlemen. As soon as Huygens's precocious genius shot its first buds, he was admitted with demonstrative honour to the choicest circles of virtuosi ; the poor Cambridge scholar had to be content with the less-glamorous, but perhaps more substantial esteem of plain people like himself, engrossed in the business of trade and administration, but compensating the lack of elegance in their intellectual demeanour by seriousness of purpose and a keen sense of reality.

It is highly instructive to wateh how John Collins puts young Newton's mathematical skill to the test by proposing to him problems of annuities and related algebraic questions : of such obscure birth is modern. analysis ! The next theme, recurring through a great part of the volume, is the construction of a reflecting telescope; it is in this work as well as in their mathematical research that Newton and Gregory followed parallel ways, of which they became acquainted through their common correspondent, the always friendly, sometimes officious Collins.

Then comes the dominant feature of the period spanned by the volume under review, the great work on the theory of colours and the nature of light, which is here very fully documented. We may follow in minute detail the famous controversy to which Newton's investigations gave rise, so startling did his new method of reasoning by induction from experiment appear even to men like Huygens and Hooke. However, a careful reading of Hooke's objections, now for the first time available in extenso (I allude to the important new document No. 71), reveals that the latter had spotted a real weakness in Newton's argument, namely, his too rigid assertion of the permanent character of the homogeneous coloured rays. In this light, Newton's famous conciliatory letter to Hooke (No. 154), which has hitherto been presented as an example of generosity, cannot fail to strike one, on the contrary, as unpleasantly condescending and arrogant. Without denying Oldenburg's part in embittering the quarrel, one feels that Newton's own obstinacy and unwillingness or incapacity to understand Hooke's point are amply sufficient to account for it.

It is well known that the conception of universal gravitation goes back to the same period of Newton's life, when, as he will later declare, "I was in the prime of my age for invention". Yet, from this time we have only one document (No. 117) of extreme interest, recently brought to light by Dr. A. R. Hall : it is the first appearance of the mightiest theme in the Newtonian symphony, which will unfold itself throughout the further, eagerly awaited instalments of this magnificent publication. L. RosenfeLD

\section{ASTROPHYSICS OF NON-STATIC SYSTEMS}

Moving Envelopes of Stars

By Prof. V. V. Sobolev. Translated by Sergei Gaposchkin. Pp. xiv +106 . (Cambridge, Mass.: Harvard University Press; London : Oxford University Press, 1960.) 38s. net.

THE astrophysicist trying to infer the development of astronomical systems from the available observations is generally like someone trying to imagine the story of a film from a few 'stills' shown outside a einema. Most astrophysical theory is concerned with such an exercise. However, the astrophysicist is occasionally afforded the advantage of a 'trailer', when he actually sees some change occurring in the heavens as in a nova eruption or in cases of matter obviously streaming away from stars. But a trailer is usually concerned with the more colourful episodes in a story, and a special skill is needed for correctly imagining the whole film from it. The present book is concerned with the analogous exercise in astrophysical theory.

The contents of the book, however, are easy neither to describe nor to assess. This is mainly because, as Prof. Sobolev says, he leaves the general account of the subject to be found in text-books and here presents only his own results, seemingly in much the form in which they were originally published in Russian journals. At any rate, he states the purpose very clearly as "to give a theory of radiative equilibrium of a moving medium and to apply it to tho moving envelopos of stars. Becauso of the breadth of the problem, only the basis of the theory and its most immediate applications are given hore".

The account is concise and free from unnecessary complications. In fact, it is so concise that only someone currently working in the field is likely to be able to appreciate its full value. But even someone such as myself whose ventures into the field wero some time ago, can realize that Prof. Sobolev has made important advances in the theory and its applications, and that Dr. Gaposchkin has done good service by making them better known by translating them into English.
W. H. MCCreA 